\title{
Sur Durkheim, l'Afrique et le Japon. À propos de trois thèses de doctorat
}

\section{Giovanni Busino}

\section{(2) OpenEdition}

\section{Journals}

Édition électronique

URL : http://journals.openedition.org/ress/368

DOI : $10.4000 /$ ress.368

ISSN : 1663-4446

Éditeur

Librairie Droz

Édition imprimée

Date de publication : 1 mars 2004

Pagination : 45-61

ISBN : 2-600-00941-8

ISSN : 0048-8046

\section{Référence électronique}

Giovanni Busino, "Sur Durkheim, l'Afrique et le Japon. À propos de trois thèses de doctorat », Revue européenne des sciences sociales [En ligne], XLII-129 | 2004, mis en ligne le 05 novembre 2009,

consulté le 22 avril 2019. URL : http://journals.openedition.org/ress/368 ; DOI : 10.4000/ress.368 


\section{Giovanni BUSINO}

\section{SUR DURKHEIM, L'AFRIQUE ET LE JAPON. À PROPOS DE TROIS THÈSES DE DOCTORAT}

Pour quelqu'un qui ne se range pas parmi les sociologues durkheimiens, la lecture de cette thèse, alimentée par les options sociologiques de Philippe Besnard et par ses connaissances étendues de l'œuvre du fondateur de la sociologie française, est à la fois exaltante et éprouvante ${ }^{1}$.

Exaltante car son auteur est arrivé à ne jamais séparer l'intelligence critique, l'audace de la pensée, la rigueur de l'argumentation, d'un certain bonheur d'expression, d'une érudition solide, d'une maîtrise certaine des sources primaires et secondaires. Exaltante car son auteur nous fait découvrir à chaque page des problématiques anciennes mais reformulées, de façon lucide et perspicace, autrement, et cela sans s'appesantir sur l'herméneutique courante et sans recourir à la polémique et/ou aux règlements de comptes rituels dans ce genre d'études. Exaltante aussi car cette thèse est si bien construite qu'aucune couture n'est plus ostensible. En effet, les trois parties (Histoires, Auto généalogies, Thèmes) se croisent, s'imbriquent parfaitement et transfigurent l'ontologie, la logique, les ressorts culturels, les débats scientifiques, les stratégies d'opposition en pièces constitutives de la charpente philosophique de l'œuvre durkheimienne.

Thèse éprouvante, disais-je aussi, parce que le lecteur se répète presque à chaque page: mais pourquoi n'avais-je pas pensé à cela? Pourquoi n'avais-je jamais douté des thèses selon lesquelles l'œuvre de Durkheim formalise le divorce de la sociologie avec la philosophie, qu'elle ne possède pas une solide cohérence interne, que ses concepts sont ambigus? Un exemple de cette ambiguïté? Si les faits sociaux ne sont pas assimilables ontologiquement à des choses, comment peuvent-ils être des choses au même titre des choses matérielles encore que d'une autre manière?

Me serais-je égaré, au cours de mes lectures durkheimiennes, aussi irrémédiablement et pendant les quelques quarante années d'enseignement de l'histoire de l'analyse sociologique?

Quoiqu'il en soit, l'excitation intellectuelle provoquée par la lecture de cette thèse prouve, en tout cas, que la prouesse de Monsieur Paoletti est originale et remarquable, évidemment louable et méritoire, et qu'elle non moins évidemment

Giovanni Paoletti, Thèse pour le doctorat en sociologie: Emile Durkheim et la philosophie. Histoire, généalogies, thèmes. Soutenance du 25 février 2003, Paris, Institut d'études politiques, rue des Saints-Pères, Salle François Goguel. (Jury: Borlandi, Busino, Colas, Merllié, Steiner). Le directeur et l'inspirateur de cette thèse a été Philippe Besnard, dont les recherches durkheimiennes ont nourri les options sociologiques du candidat. 
m'a vivement questionné, suscité en moi des doutes, des interrogations, des perplexités.

Je n'ai ni de critiques ni de réserves à adresser à l'auteur de cette thèse, que je juge excellente. Je voudrais seulement avoir quelques éclaircissements et des explications à propos des questions que je me suis posé sans arriver toutefois à bien les formuler. Les élucidations faciliteraient peut-être l'intelligibilité appropriée de ces pages et aideraient à leur bonne compréhension.

Pour des raisons de temps, je ne pourrai pas suivre le même ordre de présentation de Monsieur Paoletti, ni glaner tout son champ, par ailleurs à la moisson abondante et opulente. Ce dont je m'excuse d'avance. Le manuscrit de la thèse est arrivé à Genève le 24 janvier et j'ai eu très peu de temps pour l'analyser en profondeur et pour faire les contrôles et les vérifications d'usage.

Tout d'abord, une question préliminaire, de méthode, qui m'est venue à l'esprit au fil de la lecture de ces pages. La voici: Peut-on saisir véritablement la cohérence de l'œuvre durkheimienne, ses restructurations successives, ses relations aux problématiques ambiantes, à la conjoncture idéologique et théorique de son temps, en triant, en rassemblant des fragments, en nouant ensemble des fils ténus, en traitant des écrits, rédigés entre 1884 et 1915, comme un opus, un système signifiant clos et où les théories deviennent parfois des essences données? Est-il légitime de présumer que le rapport de l'auteur à l'histoire est configurable sur le mode de l'opposition entre un projet expressif et des obstacles à l'expression? Quid du projet de Durkheim, au lendemain de son retour d'Allemagne, de constituer une science nouvelle, de lui donner un statut universitaire, mais surtout de revitaliser la pensée française débilitée après le désastre de 1871, de la mettre à la hauteur de la grande culture allemande?

Les premières 118 pages de la thèse sont assurément les plus personnelles et les plus originales. L'analyse de contenu des occurrences du mot philosophie est réalisée parfaitement et élégamment. Ces pages ont beaucoup excité ma curiosité et donné à penser. Elles dissèquent, très doctement, de problématiques que nous sociologues de base avons pris l'habitude de négliger ou d'oublier alors que les philosophes des sciences sociales, surtout anglo-saxons, les étudient attentivement. Mais qu'est-ce qu'elles nous disent, ces 118 pages, à propos des fondements philosophiques de la sociologie de Durkheim?

Dans ces très belles pages Monsieur Paoletti revendique l'opportunité d' «essayer de concevoir la philosophie 'comme une chose'». Je reviendrai sur cette notion de «chose» tout à l'heure, mais pour le moment je voudrais seulement noter que les caractéristiques attribuées généralement à cette «chose», à la philosophie, depuis Aristote jusqu'à nos jours, ont été toujours bien délimitées. On peut les résumer rapidement ainsi: 1.la philosophie traite de la nature et de la validité du savoir; 2.elle détermine la nature des procédés fixant la validité des connaissances; 3 . elle indique où le savoir valable doit pointer et à quoi il doit servir. Les définitions données par Durkheim peuvent toutes être ramenées à l'une ou l'autre de ces conceptions.

On sait que Kant est partisan de la première de ces trois conceptions. Il oppose, dans la Critique de la raison pratique, la méthode analytique de la philosophie à la méthode synthétique de la mathématique. La connaissance philosophique, basée sur l'intuition sensible, sur des objets déjà donnés, est une connaissance de concepts, tandis que la connaissance mathématique, fondée sur l'intuition pure de 
l'espace-temps, est une construction de concepts. Ces derniers aident à trouver les causes ou les raisons des phénomènes, à déceler la rationalité cachée derrière les apparences.

Durkheim se réclame, volontairement ou involontairement, consciemment ou inconsciemment, de cette théorie de la connaissance, mais il y apporte une touche personnelle. Pour lui il s'agit de retrouver les «formes élémentaires», les «formes simples », les «formes primitives» des phénomènes, afin de dévoiler le latent dessous le manifeste, le caché derrière l'apparent. A-t-il subit l'influence des reélaborations kantiennes de Renouvier, de Hamelin, de Boutroux?

Les arguments favorables à la réponse affirmative me semblent solidement établis mais ils ne balayent pas tous mes embarras. Je suis convaincu que le rationalisme, théorique et pratique, de Durkheim a une coloration trop particulière et que même son positivisme est une conséquence de ce rationalisme. C'est bien ce rationalisme qui l'oblige à répéter que son option naturaliste est un choix purement épistémologique. Certes, les objets des sciences sociales ont une spécificité propre mais leur méthode est identique à celle des sciences de la nature. Les unes et les autres s'enracinent dans le même principe d'intelligibilité, la causalité, output d'une induction légitime du postulat empirique, celui de «la nature des choses », fondement premier d'une loi objective par elle-même et en elle-même. Mais si la relation causale est comme une loi inhérente à la nature des choses, le concept, scientifique ou vulgaire, ne peut être qu'une copie du réel, de l'ordre naturel des choses, où effectivement tous les phénomènes sont liés par des rapports nécessaires.

Qu'est-ce que cet ordre objectif des choses, qu'est-ce que la «nature des choses »? Durkheim a donné toujours la même réponse: «...ce n'est pas à nos préjugés, à nos passions, à nos habitudes que doivent être demandés les éléments de la définition qui nous est nécessaire; c'est à la réalité même qu'il s'agit de définir». «La chose s'oppose à l'idée comme ce que l'on connaît du dehors à ce que l'on connaît du dedans.»

La notion de chose est opposée à celle d'idéologie, pour laquelle tout idée correspond à un fait. Etre réel, donc objectif, est une propriété de l'appartenance à la nature des choses, à un ensemble de données, extérieurs à nous, obéissant à la loi de causalité impersonnelle. Cette notion de «la nature des choses» est stratégique dans la formulation du système théorique de Durkheim, elle conditionne tout son vocabulaire. Ce n'est donc pas un hasard qu'il parle de fait social, moins fréquemment de phénomène social, presque jamais de relation ou d'activité sociale; c'est n'est pas une trouvaille rhétorique s'il répète inlassablement: "Traiter des faits d'un certain ordre comme des choses, ce n'est pas les classer dans telle ou telle autre catégorie du réel; c'est l'observer vis-à-vis d'une certaine attitude mentale.»

Cette notion de «nature des choses » n'a rien de pareil ni chez Kant ni chez les néo-kantiens ni non plus chez Comte ou les philosophes positivistes français. Par contre, on la retrouve chez les jusnaturalistes du XVIII et chez les juristes allemands du XIX ${ }^{\mathrm{e}}$ siècle, par exemple chez Rudolf von Jhering (1813-1892) qui oppose la connaissance scientifique, basée sur la conceptualisation, à la connaissance commune, dépourvue de rigueur, de finesse et de précision. La connaissance scientifique fait coïncider ce qui est incertain et confus dans la connaissance commune avec la réalité objective extérieure. Entre les deux formes de connaissance, la scientifique et l'ordinaire, il n'y a qu'une différence de degré. 
Durkheim affirme des idées semblables dans les Règles...: «Ce n’est pas sans doute que le concept vulgaire soit inutile au savant: il sert d'indicateur. Par lui, nous sommes informés qu'il existe quelque part un ensemble de phénomènes qui sont réunis sous une même appellation et qui, par conséquent, doivent vraisemblablement avoir des caractères communs; même, comme il n'est jamais sans avoir eu quelque contact avec les phénomènes, il nous indique parfois, mais en gros, dans quelle direction ils doivent être recherchés. Mais, comme il est grossièrement formé, il est tout naturel qu'il ne coïncide pas avec le concept scientifique institué à cette occasion.»

La réalité est le monde matériel concret, c'est «la nature des choses » extérieure source des explications causales. Possède-t-elle également une signification externe? Le cas échéant, de quelle façon agit-elle sur la compréhension du sujet? Peut-on affirmer qu'une signification est la cause d'une autre alors que tout indique que les significations s'entraînent les unes aux autres selon un mode de liaison que Jean Piaget qualifierait « de nature plus noétique ou inférencielle que causale »? Pour quelles raisons la distinction entre l'ontologie et la logique est si incertaine, jamais nettement tranchée?

Parce que, selon moi, Durkheim n'arrive pas à établir une démarcation précise entre la cause et l'implication. Ancrées dans la contrainte, elles, la causalité et les implications normatives, sont données dans une proportion constante et conçues comme un seul tout. La synchronie est absorbée ainsi dans la diachronie alors que les relations de causalité sont liées à une succession dans le temps pendant que celles implicatives dépendent d'un rapport nécessaire mais extemporané. En plus, si la subjectivité est variabilité, si l'ordre institutionnel ne peut pas être le fondement de l'objectivité, si une institution humaine «ne saurait reposer sur l'erreur et sur le mensonge », il en découle fatalement que la société n'est que «la nature des choses ». Si ce n'était pas ainsi, elle rencontrerait «dans les choses des résistances dont elle n'aurait pu triompher», dit-il.

En outre, si la société possède des repères constants et identiques, des formes permanentes et invariables, «un étalon constant qui est toujours à la portée de l'observateur et qui ne laisse pas de place aux impressions subjectives et aux observations personnelles », elle est, évidemment, productrice de faits sociaux, de représentations «d'une autre nature et d'une autre sorte que les états de la conscience individuelle», de concepts qui «expriment la manière dont la société se représente les choses».

Ces questions, je l'avoue humblement, ont toujours alimenté mes réticences à l'endroit de la pensée de Durkheim. Peut-être que mon orthodoxie webernienne les a biaisées et qu'une très longue pratique de Pareto les a aggravées.

D'autres questions, il me semble, restent sans démonstrations satisfaisantes. A titre d'exemplification ou d'illustration en voici une ou deux: Si l'on écarte l'explication biologique causale, si l'on rejette le principe d'économie naturelle, si l'on refuse le rapport interne du sujet connaissant à l'objet ainsi que l'universalité subjective et la validité commune, comment peut-on éviter les raisonnements aporétiques? Dans l'impossibilité de s'écarter de ce piège, les universaux, soit au sens ontologique que logique, et la réduction de la validité subjective à la crédibilité, deviennent à la fois concepts, signes et signifiants, le réalisme et le nominalisme ne sont plus nettement discernables tandis que le conceptualisme, délesté de sa fonction logique de prédication, ne détermine plus, si non peut-être de façon floue, la notion de limite. 
L'impétrant écrit des pages remarquables sur la limite et le lien social, sur l'homo duplex. Avec finesse et nuances, il a noté que chez Durkheim il y a deux conceptions alternatives de la limite, celle par équilibre et celle par juste milieu, et qu'aucune de ces deux conceptions ne peut être considérée satisfaisante. Mais pourquoi le terme de limite prend chez Durkheim des acceptions différentes, pourquoi il en fait des usages si disparates par rapport à la tradition kantienne et à la logique (où il renvoie aux notions de fonction, de continuité, de dérivée, de série et de suite)? L'auteur suggère implicitement, timidement, que les inconvénients du terme limite sont sérieux et dans une certaine mesure qu'ils fragilisent les théories de l'intégration et de la régulation sociale. Est-ce que ces deux théories ont véritablement besoin de la notion de limite?

En faisant de la pensée collective une pensée impersonnelle, immuable, universelle, l'unique source de vérité et d'objectivité, l'expression primordiale de la nature des choses, la logique ne peut être produite que par la collectivité, elle est vraie parce que collective et collective parce que vraie. La société symbolise l'objectivité et les concepts logiques produits par la collectivité trouvent dans la collectivité les «garanties d'objectivité». Raisonnement en boucle, pétition de principe, circularité de la définition?

Jean Piaget nous a appris que la logique n'est ni une qualité donnée ni un état, qu'elle est l'expression d'un processus, qu'elle est une théorie des opérations déductives, effectuées par l'individu, ou échangées grâce au langage. Il ne se lassait jamais de redire: «La logique est le miroir de la pensée et non l'inverse. La logique n'est pas coextensive à l'intelligence, mais consiste en l'ensemble des règles de contrôle dont use l'intelligence pour se diriger elle-même.» Il y a une logique des opérations concrètes et une logique des opérations formelles ou propositionnelles, une logique statique et une logique opératoire. La prise de conscience d'une relation, ou d'une règle, doit être apprise sur les plans de l'action et de la pensée en tant que transformations mobiles et réversibles. Les sciences cognitives, et notamment la sémantique cognitive ne nous offrent aucune aide pour soutenir l'idée que la logique, la vérité et l'objectivité sont produites par la société.

Il en va de même pour les catégories, lesquelles, selon Durkheim, «viennent de la société », «les choses qu'elles expriment sont sociales», et elles «ne peuvent devenir conscientes que dans et par la société». En résumé, toujours selon Durkheim, toutes les catégories (perceptuelles, conceptuelles, primaires, régulatrices, essentielles, réelles et formelles) sont sociales. Mais elles ne sont pas définies par les notions de totalité, de relations, d'idées et de valeur, ni par leurs fonctions explicatrices (causalité, réalité, temps et lieu, justification des règles) ni par leurs fonctions implicatives (classification, nom, nombre), ni même par leurs relations logiques (l'élaboration déductive indéfinie). Opérateurs de savoirs, formes primitives de classification, les catégories durkheimiennes ont une origine sociale mais ne sont pas sociales de façon interne. Pour le sociologue et pour le logicien cela est un paradoxe effrayant. Steven Collins a essayé, en 1985, de clarifier la question en remontant aux origines philosophiques des catégories durkheimiennes. Il a découvert que Renouvier et Hamelin, influencés par les doctrines hégéliennes du changement et du développement, avaient une vision historique des catégories de la pensée, alors que Durkheim s'est voué à l'élargissement des catégories kantiennes et qu'il en a fait un principe universel d'organisation 
conceptuel, indépendamment des procédures d'accès aux entités rassemblés. Collins donne une grande importances à une affirmation comme celle-ci: «Il existe, à la racine de nos jugements, un certain nombre de notions essentielles qui dominent notre vie intellectuelle; ce sont... les catégorie de l'entendement». «Elles sont comme les cadres solides qui enserrent la pensée; celle-ci ne parait pas pouvoir s'en affranchir sans se détruire». Catégories innées, données de l'extérieur? Comment et pourquoi elles ont été élaborées?

Je n'ai pas besoin d'en dire davantage car toute cette très difficile problématique a été approfondie, en France, par des auteurs dont les études se trouvent dans le volume édité par Fradin, Queré et Widmer, paru en 1994, et intitulé L'enquête sur les catégories. De Durkheim à Sacks. Cette même problématique a été revisitée par Conein, Trépos et Thévenot dans le livre Sociologie et Connaissance. Nouvelles approches, édité en 1998 par Borzeix, Bouvier et Pharo. Toutes ces études montrent bien que les catégories durkheimiennes ne sont pas des concepts mais des fonctions régulatrices, qu'elles sont plutôt un processus de mise en relation d'un univers extérieure avec une activité intérieure ordonnant et déterminant un ensemble de concepts (objets, espace, causalité, temps). D'ailleurs, c'est ce processus qui rend possible la pensée, la capacité d'évoquer par un signe ou une image symbolique l'objet absent ou l'action non encore accomplie. Piaget a démontré que les catégories rendent possibles les représentations «à partir seulement du moment où aucun indice perçu ne commande la croyance en la permanence, c'est-à-dire à partir de l'instant où l'objet disparu se déplace selon un itinéraire que le sujet peut déduire mais non percevoir». Dans ces conditions «La représentation consiste... soit à évoquer les objets en leur absence soit, lorsqu'elle double la perception en leur présence, à compléter leur connaissance perceptive en se référant à d'autres objets non actuellement perçus. Elle introduit donc un élément nouveau, qui lui est irréductible: un système de signification...». Comment concilier ces nouvelles approches de la catégorisation et de la représentation sociales avec les positions de Durkheim pour lequel le concept de représentation est vide? En effet, pour le sociologue français la représentation individuelle ne peut pas être ramenée à l'activité cérébrale qui la rend possible tandis que la représentation collective est irréductible à la somme des représentations des individus qui composent une société. Or représenter un état (ou une chose) n'est jamais un dédoublement ou une reproduction; il faut le reconstituer, le retoucher, le changer de cadre. Cette activité intellectuelle est une œuvre de communication et de groupe, elle se réalise au cours de processus d'échanges et d'interactions, individuels ou groupaux. Elle n'est ni une structure cognitive spécifique ni une classe de connaissances collectives. Ce mot collectif, qui s'applique à tout, finit, par ailleurs, par ne désigner plus rien. Serge Moscovici l'a fort bien établi dans ses travaux sur les représentations individuelles et les représentations sociales. À raison il a écrit: "Il s'agit de comprendre, non plus la tradition mais l'innovation, non plus une vie sociale déjà faite mais une vie sociale en train de se faire », non plus des sociétés dites primitives mais des «mécanismes psychiques et de communication produisant un phénomène spécifique».

Sur ces points l'impétrant est, il me semble, hésitant, bien qu'il connût fort bien la bibliographie en la matière et qu'il la tressât avec intelligence et lucidité.

J'ai particulièrement apprécié, dans la troisième partie de la thèse, le jugements sagaces, les rapprochements subtils, les remarques pénétrantes sur un sujet 
au cœur des sciences cognitives et des logiques naturelles, sur un sujet que la psychologie sociale, et plus particulièrement Serge Moscovici, Jean-Blaise Grize, Denise Jodelet, Claudine Herzlich et tant d'autres, ont redéfini et déterminé de fond en comble depuis la fin des années '70. M. Paoletti aurait pu utiliser davantage leurs travaux afin d'étayer mieux ses analyses.

Pour finir, j'aimerais dire quelques mots sur un tout petit nombre de points de la troisième partie de la thèse. Monsieur Paoletti analyse avec dextérité les débats sur la notion de croyance en France de l'époque du Traité de psychologie rationnelle...de Renouvier à celle des Formes élémentaire de la vie religieuse, le chefd'œuvre de Durkheim. Par contre, les pages sur l'épistémologie durkheimienne de la croyance ne m’ont pas convaincu. Peut-on véritablement corréler les représentations collectives et les croyances? Comment prouver que les croyances consistent en des représentations, que ces représentations sont collectives et qu'elles sont vraies d'autant plus si elles sont en harmonie avec les croyances? Je lis à la page 215: «Croyance et représentations se définissent réciproquement.»

J'ai exprimé déjà mes perplexités à propos de la notion de représentation. Celle de croyance soulève mes réticences et me plonge dans le scepticisme. Si nous admettons l'hypothèse qu'une proposition peut être tenue pour vraie si elle est accompagnée d'une croyance, nous ne pouvons plus affirmer que la croyance est une disposition à agir d'une certaine façon devant certains objets. Un sujet croit à une proposition, donne son assentiment à une doctrine, adhère et accepte des faits vraisemblables ou possibles, selon le genre, le nombre et la résistance des obstacles qu'il est disposé à vaincre pour continuer son action selon cette proposition, cette doctrine, ces faits. Ceci étant, je ne suis pas sûr qu'une croyance soit nécessairement une connaissance. Peut-être que l'impétrant, au fil de la discussion, arrivera à me convaincre du contraire.

En ce qui concerne la théorie du symbolisme, Monsieur Paoletti a des excellentes raisons de ne pas ramener la notion de symbole à celle de représentation, de soutenir avec force que les symboles sont irréductibles aux représentations, et de nous rappeler que Durkheim parle aussi d'emblème et d'image. Il fait preuve de perspicacité lorsqu'il écrit aux pages 231 et 232 : «Le symbole est une image matérielle, c'est-à-dire individuelle. Il se situe sur le plan de la vision plutôt que sur celui du langage [...] les sensations individuelles ne sont pas l'élément sur lequel se fonde le symbole. Mais un moyen pour atteindre quelque chose d'autre.» À la page 235, il ajoute: «Le symbole est une image parce qu'il rend visible l'invisible; c'est une image simple, parce qu'il est censé rendre compréhensible le complexe; il est concret, parce que les sentiments doivent se fixer sur lui; il est matériel, parce que sans extériorité il n'y a pas de société.» On pourrait être d'accord à condition toutefois de préciser que le symbole est une image évoquée mentalement ou un objet matériel choisi intentionnellement pour désigner une classe d'objets ou d'actions; à condition de reconnaître que le symbole est un assemblage, une réunion de sentiments et d'idées disparates et multiples en une seule image qui les résume. Cet assemblage, chargé d'affectivité, ne peut être qu'un concept très embryonnaire. Pour cette raison j'aurais aimé avoir davantage d'explications à propos de ce que je lis à la p. 242: «...les représentations scientifiques (ou du moins sociologiques) elles-mêmes ne sont pas, sans le recours au symbolisme.»

Mais si le symbole est individuel et motivé, il faut quelque chose d'autre pour produire un énoncé scientifique, ou sociologique, il faut le signe (au sens 
saussurien) qui est général et abstrait. Si je veux élaborer une signification, j’ai besoin à la fois d'un symbole individuel et d'un signe social. Le symbole est une condition nécessaire mais non suffisante de l'apparition des signes. Sans les signes le symbolisme n'arrive pas à attribuer la cognition et l'objectivité aux phénomènes d'interaction et de communication.

L'impétrant dit que les représentations ont une composante autoréférentielle, que l'ontologie de Durkheim est à deux niveaux (individu et société, réalité et représentation), que la réalité extérieure existe bien que sa représentation soit problématique. Il ajoute qu'il n'y a pas un Durkheim positiviste et un Durkheim idéaliste, et conclut son travail ainsi: «...on préférera saisir la continuité d'une recherche qui, à partir de la question de la forme de la représentation, s'est tournée vers celle de son objet.»

Cette thèse est un effort admirable et courageux d'ouvrir l'œuvre durkheimienne à cette perspective. J'ai admiré cet effort mais je suis resté en lecteur incrédule. J'ajoute que cette thèse occupe une place de choix, plus qu'honorable, dans ce courant intellectuel dont le grand et beau livre de Philippe Besnard sur l'anomie est la table des lois et les travaux de Pickering et de Stedman Jones les évangiles. Ces travaux (et cette thèse va occuper une place stratégique dans ce courant) nous donnent à voir un autre Durkheim, plus ouvert, plus nuancé, moins doctrinaire. Ces travaux ne me font pas devenir durkheimien, mais ils confortent ma décision de m'éloigner des thèses que mon maître Jean Piaget avait assénées dans Sagesse et illusions de la philosophie; ils me libèrent définitivement du préjugé anti-durkheimien qu'a été le mien dans le passé. Ils me persuadent pour de bon qu'il n'y aura jamais une véritable science sociologique sans une bonne collaboration avec la philosophie et l'histoire.

La lecture de cette thèse, de ce point de vue, offre une contribution très appréciable, très méritoire, très honorable. Et pour ces raisons son auteur a droit à notre gratitude.

Cette thèse ${ }^{2}$ a le mérite de dépasser les objets courants de la sociologie de la littérature. Les textes littéraires y sont pris au sérieux et par delà les thèmes communs aux écrivains retenus, l'auteur a essayé de dévoiler, par étapes raisonnées, chez des lettrés colonisés et chez des romanciers négro-africains, depuis la fin du $\mathrm{XIX}^{\mathrm{e}}$ siècle jusqu'aux années ' 80 du $\mathrm{XX}^{\mathrm{e}}$ siècle, l'homologie de leurs rapports à l'Afrique. Ceci est fait avec une audace intellectuelle modeste mais ferme.

Cette thèse jette, en effet, quelques lumières sur le rapport des écrivains à l'Afrique, sur ce rapport complexe, compliqué, difficile, varié et singulièrement disparate. Jusqu'ici nous ne disposions que de rares matériaux pour bien cerner la problématique et de quelques éléments nous conviant à un début d'explication. Même les récits foudroyants de Yambo Ouologuem dans Le devoir de violence, paru en 1968, qui nous paralysent d'étonnement et nous plongent dans la souffrance; même ses descriptions de l'asservissement pusillanime aux blancs, du

Mathias D. Dossou, Thèse de doctorat en sciences sociales: Figures d'intellectuels occidentalisés dans le roman africain d'expression française. Soutenance du 2 mai 2003. Lausanne, Institut d'anthropologie et de sociologie de l'Université (Jury: Berthoud. Busino, Monnier et Mouralis). 
mépris de tous les droits, de la vie humaine, des razzias, des exactions, des meurtres, toutes ces descriptions sont restées sans suite.

On pourrait citer les livres d'autres écrivains, par exemple celui de Aminata Traraoré, Le viol de l'imaginaire, décrivant le drame du continent africain en tant que reflet de l'ordre du monde, exorcisant la panoplie des mots creux qu'elle appelle les mots-clés de l'ordre. On pourrait aussi rappeler que la situation des écrivains africains de langue anglaise est encore plus critique. Charles R. Larson dans son The Ordeal of the African Writer fait l'inventaire des calvaires de ces écrivains, dont les stations de la via crucis sont la prison, l'exil, la folie, la mort. Et on pourrait établir un inventaire analogue pour les écrivains de langue française d'Afrique noir et du Magreb.

Est-ce que retracer les contours de ce champ littéraire émergeant avec beaucoup de peine, pourrait nous aider à comprendre ce rapport des écrivains à l'Afrique? Des critiques en doutent. Peut-être pour cette raison qu'il a été écrit récemment que les intellectuels africains ne savent pas, ne veulent pas, ne peuvent pas «fonder une écriture qui traque toutes les forces qui, de près ou de loin, mettent cette vie en danger.» L'écrivain camerounais Patrice Nganang affirme, dans l'article L'écrivain africain et le syndrome rwandais, "Le Monde», 15 janvier 2003: «[....] tant que l'africain, écrivain ou pas, n'aura pas imaginé dans tous ses contours ce qui est un être vivant, un homme, soit, et qui a des droits, qui a des devoirs, qui a des limites aussi, mais qui n'a vraiment pas le droit de mourir simplement comme ça, il ne se sera pas encore arraché du long sommeil du dormeur de Goya. Et son réveil, même brutal, devant les cauchemars qui ont lieu sur son continent, ne pourra jamais qu'être trop tardif.»

La thèse de Monsieur Dossou est une bonne contribution à l'éclaircissement de ce rapport bien qu'il ait repéré, c'est vraie, uniquement les thèmes traités par les écrivains sélectionnés et qu'il n'ait pas pris en compte ce qui crée l'œuvre littéraire, l'écriture, ni donné de l'importance aux conditions sociales des actes créateurs, ni traité les écrivains en simples producteurs et les œuvres en tant que produits proposés sur un marché. Il s'agit, certes, d'un choix discutable mais il lui a évité le risque de sombrer dans le réductionnisme sociologique. Cela a quelques avantages substantiels. Les raisons en sont évidentes. Par définition, le point de vue sociologique implique que la signification d'un texte ne s'épuise pas dans le texte lui-même. Le texte seul, défini par sa seule textualité, ne peut pas dire tout. Mais le danger est grand d'aboutir, par un effet de balancier, à ce qu'on oublie le texte, que l'on évacue soit au profit d'une sociographie du monde de l'édition, des conditions du marché, du fonctionnement des institutions littéraires ou académiques, soit d'un discours, déconnecté de toute réalité concrète, sur les structures sociales ou les rapports de domination.

L'impétrant a contourné, donc, ces difficultés au prix d'une esquive des certains problèmes fondamentaux, parmi lesquels celui des critères de définition de l'intellectuel africain me parait le plus important. Le rôle civique, l'engagement, l'opposition aux valeurs et aux visions du monde des groupes dominants ne sont pas des critères suffisants. Il faut, pour bien caractériser la catégorie, l'existence de degrés d'autonomie par rapport aux pouvoirs extérieurs, aux intérêts économiques et aux conventions sociales. Il faut aussi la présence d'un appareillage symbolique de déchiffrement et d'évaluation et l'existence d'une opinion publique. Existent-elles, ces conditions, en Afrique? 
Monsieur Dossou a repris quelques thèses de Goody, notamment celles argumentées dans «Des intellectuels dans les sociétés sans écriture?», beau texte où il démontre qu'il n'y a pas de sociétés humaines dépourvues d'activités intellectuelles créatrices et même d'intellectuels. Et il a bien fait. Même C. Lévi-Strauss et P. Guillon ont démontré, en son temps, que l'avènement de l'écriture n'exprime pas nécessairement un accès à un niveau supérieur de culture. Cependant l'écriture est une forme de présentation du savoir; elle en détermine aussi le contenu.

Dans l'univers écrit la parole devient incertaine et inefficace, le secret y est ruiné; elle ne véhicule plus les ésotérismes, les interdits, le sacré ineffable. Par contre, dans l'univers oral la valeur du verbe (parole, rythme, symbole, complexité dans l'art d'exprimer les nuances) est absolue. L'argumentation orale est fragilisée par l'inattention et l'oubli; elle enseigne mais par répétitions, allitérations, rythmes, métaphores, allégories, énigmes. Sa fonction poétique est indubitable; celle critique insuffisante. La production des énoncés oraux est une recréation; la transmission inaltérée des contenus y est impossible; les contenus sont réaménagés en fonction des circonstances, des intérêts, des goûts du moment, des fins différents ou de visées contraires. Et cela a des répercussions profondes sur la mémoire et l'apprentissage.

Goody a écrit un texte magistral sur les fonctions de la mémoire et de l'apprentissage dans les sociétés avec et sans écriture, sur les relations déroutantes entre les cultures de l'oralité et les cultures de l'écriture, notamment en Afrique occidentale. Tous les travaux récents n'ont pas modifié les conclusions que l'anthropologue anglais avait ébauchées, en 1984, dans Cuisines, cuisine et classes sociales où il essaie de trouver une réponse à la question «pourquoi une grande cuisine n'est pas apparue en Afrique?», conclusions que par la suite il a admirablement synthétisé dans le grand livre de 1993 Entre l'oralité et l'écriture.

L'analyse de ces dissemblances entre l'oralité et l'écriture aurait fait comprendre beaucoup mieux, je crois, pourquoi l'homogénéisation des populations a été si lente ainsi que la progressive différenciation des couches et des classes sociales. Elle aurait mis en évidence les profondes mutations induites par l'Islam, et pas seulement par l'occidentalisation et la modernisation.

J'ai apprécié tout particulièrement la deuxième partie de la thèse où sont décrites, avec précision et minutie, les tribulation des romanciers qui ont tenté de donner un sens à leur nouvel univers, de produire une synthèse entre des traditions pour eux encore significatives et celles de la sécularisation technicienne, entre les valeurs du passé et celles solliciteuses de la modernité occidentale.

Le rapport à l'Afrique et les orientations éthico-politiques de ces romanciers ne me paraissent pas totalement analogues. Et pour cause. Comme tous les comportements humains, ce rapport et ces orientations comportent des significations multiples.

La thèse relève des similitudes thématiques et suggère que le rapport personnel de chacun de ces écrivains à son Afrique, aux traditions, a facilité un certain type d'action, a introduit les lecteurs au cosmos social et par là il a permis certaines anticipations. Toutefois l'impétrant note, ici et là, et parfois de façon très pertinente, que le rapport personnel de ces écrivains africains d'expression française reste ambivalent. Il sert des buts différents, il n'a pas d'essence fixe et invariante. Dans ses applications il est fluet et ses délimitations restent souvent vagues. 
Ailleurs Monsieur Dousse écrit que ces écrivains affichent les mêmes valeurs d'appartenance, de référence et d'authentification. Il dit aussi que l'appartenance renvoie à la multiplicité des groupes sociaux qui prennent en charge les Africains. Les classes d'âges, les rôles, les status, les fonctions développent entre les sujets des liens de solidarité, fondement de l'appartenance. Mais comment concilier l'ambivalence avec ces valeurs?

J'aurais aimé en savoir un peu plus sur ces questions d'appartenance, de référence et d'authentification. A quoi renvoie, par exemple, la valeur d'authentification? Aux mythes, aux ancêtres, à l'exaltation collective, à la conception du temps, du passé, du présent, de l'avenir?

Une réponse à cette question m'aurait permis de mieux comprendre la nature de l'identité profonde de ces intellectuels occidentalisés, identité dont l'auteur de la thèse parle constamment mais qu'il évite toutefois de définir de façon convaincante.

Encore une observation. Je trouve que Monsieur Dossou sous-évalue la négritude. Elle a été une idéologie puissante pour contrer l'idéologie coloniale et pour l'avènement de certaines formes de nationalisme culturel. Chez Senghor elle est aussi un flamboyant style littéraire qui donne une forme séduisante aux conduites de compensation et/ou de réaction à la frustration coloniale. En exaltant la personnalité du Noir, en protestant contre l'ignorance de la réalité nègre de l'homme occidentale, l'idéologie de la négritude, notamment dans la phase de transition du colonialisme à l'indépendance, a permis de retrouver, de défendre, d'épurer et de repenser les spécificités et les particularités des sociétés et des systèmes culturels de l'Afrique. En tant qu'idéologie elle a eu une fonction essentielle; en tant que philosophie et doctrine littéraire, la négritude est tombée dans l'obsolescence lorsque ses fonctions sont devenues périmées. L'histoire lui a déjà rendu son dû. Il aurait été souhaitable que vous en fassiez autant.

D'où vient l'intérêt de cette thèse? De l'information neuve qu'elle produit? Sans doute. Cette nouveauté est-elle affaire de contenu d'information? En partie, certainement. Et pourtant... Il y a dans le style de Mathias Dossou quelque chose de discret, d'allusif, d'interrogatif qui le rend inimitable. La nouveauté de cette thèse ne provient pas du contenu mais de la relation qui s'instaure entre l'auteur et les romans élucidés. Le style est en effet la marque d'une voix qui s'exprime. Et la voix appelle davantage du côté de la relation que du contenu. Elle bouscule les frontières disciplinaires traditionnelles, ouvre des perspectives fécondes et rend compte des phénomènes complexes, mouvant, irréductibles aux classifications, aux définitions conceptuelles des objets des sciences.

La lecture de cette thèse a appris beaucoup au profane de l'histoire, de la culture et de la littérature africaines que je suis. Elle a consolidé ma certitude que les nouvelles générations d'intellectuels donneront à la culture africaine la place honorable qu'est la sienne parmi les cultures de notre Planète.

Dans cette perspective je considère cette thèse une contribution appréciable et très honorable. Par conséquent je ne peux que souhaiter le foisonnement d'autres recherches dans ce genre. Elles fourniront ainsi la preuve de la vitalité, de la fécondité, du développement de la science sociale en Afrique.

Depuis 1981 Madame Maurer m'a donné à lire une énorme quantité de ses écrits, il m'a fallu parcourir je ne sais plus combien de versions de sa thèse, 
prendre connaissance de centaines de ses lettres et d'une multitude de ses prises de positions. Je l'avoue: il m'est arrivé de croire d'être en train de devenir pareil à Joseph Prudhomme, le personnage falot des deux comédies de l'écrivain et caricaturiste français du XIX ${ }^{\mathrm{e}}$ siècle, le désormais oublié Henri Monnier.

Pareillement à ce personnage, j'eus la prétention de suivre l'évolution de tous les courants intellectuels qu'elle explorait. Comme lui je crus d'entrevoir de la lumière en toutes choses. Il m'arrivai parfois de rester hésitant, incertain du parti à prendre en présence de ce qui se trouvait hors des sentiers balisés. Très impressionné par des pages où les thèses, les antithèses et les synthèses, les propositions à propos des politiques publiques en matière de consommation de drogues, de réorganisation de l'enseignement et de la recherche en santé publique, de coopération interdisciplinaire, de sociologie du lien social, et même de philosophie des relations humaines, s'amoncelaient et s'enchaînaient sans trêve, il m'a fallu faire un effort désespéré pour ne pas devenir comme Monsieur Prudhomme, niais, conformiste et sentencieux.

Je ne vais pas analyser les innombrables problématiques abordées dans cette thèse ${ }^{3}$ mais seulement quelques points à propos desquels la prudence et la vigilance doivent être de mise.

Madame Maurer a donné à sa thèse une forme assez particulière, voire inhabituelle en sociologie. Chez les sociologues on commence par définir le problème, par analyser la littérature, par formuler des hypothèses, par choisir les méthodes, et ensuite on enregistre les données, on discute les résultats en fonction de la théorie et de l'état des recherches existantes et à la fin on essaye de définir les solutions acquises et les problématiques encore ouvertes. Elle a choisi une autre approche: dire pourquoi une autre politique de la santé publique est nécessaire et comment elle doit être élaborée. Je présume que sa formation de médecin, ses expériences pratiques dans des centres médicaux de recherche, l'ont entraîné à construire cette thèse d'une manière qu'à beaucoup de sociologues peut paraître hermétique, peut-être même étrange. Certes, Madame Maurer en était consciente dès le départ et cependant elle a voulu travailler différemment, à sa guise, en son âme et conscience.

Dire que cette option pose des problèmes complexes et compliqués, ce n'est qu'une litote. Néanmoins je vais essayer, pour autant que j'en suis capable, de décortiquer la logique de ces travaux commencés il y a très longtemps, en 19811984.

Madame Maurer présume qu'il n'y a pas de société sans « défense sociale» et que le modèle japonais de défense sociale est un des plus performants. Je note en passant que la notion de défense sociale est plus commune à la criminologie et au droit qu'à la sociologie. Dans le vocabulaire de la sociologie la notion de contrôle social est plus courante. Elle indique la totalité des actions destinées à assurer, de façon formelle ou informelle, par le biais d'institutions publiques et des groupes

Milka Maurer, «Non absolument non!». Le contrôle de la criminalité, de la délinquance et de la consommation de drogues. Un succès étonnant, un espoir pour nous! La «défense sociale» 1966 1992. Un modèle de contrôle des problèmes sociaux apparu comme conséquence de l'industrialisation. Colloque du 28 janvier 2003 pour l'admission au doctorat en sciences sociales. Lausanne, Institut d'anthropologie et de sociologie de 1'Université. (Jury: Busino Dauwalder, Deschamps, Paccaud, Rey et Yoshida). 
primaires, moyennant l'intériorisation des modèles sociaux, un certain standard de conformité dans les activités individuelles et collectives. Un courant de recherche actuellement à la mode, l'ethnométhodologie, a démontré que les contrôles, même les plus intenses et les plus contraignants, même ceux exercé par les proches, sont souvent l'objet de création, d'invention, de négociation d'où une certaine instabilité, une légitimité tantôt incertaine tantôt contestée, parfois jugée contradictoire. En sociologie la notion de contrôle social est utilisée comme équivalente à celle de régulation sociale, en anglais social control.

L'auteur englobe dans la notion de «défense sociale» la criminalité, la délinquance et la consommation de drogues. La tradition sociologique, à tort ou à raison (la question reste ouverte), a toujours distingué la criminalité/délinquance de la toxicomanie. Et c'est bien à cause de cette distinction que les sociologues utilisent le concept de «contrôle social» et laissent aux juristes et aux criminologues celui de «défense sociale». Mais passons. Une fois le concept de «défense sociale »adopté, l'auteur établit un très long catalogue de ce qu'elle dénomme le modèle japonais. Ce catalogue énumère les outils dont le Japon s'est doté pour promouvoir la santé, l'éducation des enfants et assurer l'assistance aux mères en particulier et aux jeunes en général. Ensuite Madame Maurer décrit les politiques de prévention, de répression, de récupération et de réhabilitation. Dans la deuxième partie de la thèse, après avoir énuméré les performances du modèle dans la maîtrise de la criminalité, de la délinquance et de la consommation de drogues, elle suggère que les Européens devraient en tenir compte lors de l'élaboration de leurs politiques publiques. La description du modèle japonais lui sert à évaluer en retour et par contraste les modèles Européens, et notamment les pratiques Suisses. Il s'agit-là d'une forme de comparaison qui privilégie les différences mais reste indifférente à la recherche des éléments constants et à l'élaboration des types idéaux.

Les difficultés épistémologiques de ce type d'approche sont bien connues depuis Leibniz et John Stuart Mill; elles sont devenues incontournables grâce aux analyses de Durkheim, Wittgenstein et Smelser. La non prise en compte de ces difficultés rendent certaines des propositions de cette thèse invérifiables, tout au moins avec les outils dont le sociologue dispose actuellement.

Je reconnais que pour l'auteur de ce travail les problèmes épistémologiques et méthodologiques sont subalternes. Pour Madame Maurer il n'y a aucune différence entre la cognition et la décision, entre la théorie et la pratique. Elle proclame, ici et là, au détour d'une phrase, que tout savoir doit servir surtout à résoudre les problèmes concrets. Assurément, sa formation de médecin l'a habituée à concevoir la théorie comme une voie pour arriver à une thérapie. Dès lors elle concentre tous ses efforts afin d'établir l'inventaire catalogue des divers aspects du modèle japonais pour ensuite les confronter avec ceux du modèle suisse. L'exploitation qu'elle fait de la littérature grise, des entretiens avec des opérateurs japonais, de ses propres observations directes, ne m'a pas permis d'effectuer les vérifications usuelles. Il a fallu s'en remettre à l'avis du docteur Yoshida. Ce haut fonctionnaire de l'OMS, expert dans le domaine des narcotiques, a rendu hommage aux analyses méticuleuses, à l'exactitude des descriptions de cette thèse. Il a remarqué cependant que l'auteur n'a pas tenu compte des évolutions récentes, lesquelles, notamment depuis la fin des années '80, ont fragilisé le modèle japonais, modifié certaines de ses propriétés anciennes et de ce fait 
l'ont rapproché, singulièrement dans le domaine de la consommation des drogues, des modèles des pays industrialisés. MacCoun et Reuter, par ailleurs, l'ont démontré de façon incontestable dans leur livre de 2001, Drug War Heresies.

Sur la première partie de cette thèse je ne peux pas en dire davantage étant donné la singularité de la documentation utilisée. Qu'il me soit permis toutefois de constater, en passant, qu'il me paraît impropre de mettre sur le même plan les données nationales et celles des spécificités locales, à moins que ces dernières soient utilisées à titre d'illustration ou d'exemplification. Mais dans ce cas, il aurait fallu les traiter à part.

L'expertise de Monsieur Pierre Rey, directeur de la Fondation du Levant, praticiens des comportements toxicomanes, de la désintoxication des toxicos, analyse la question de l'éclairage du modèle suisse par le modèle japonais. Il retient, dans cette thèse, uniquement les parties consacrées aux toxicomanies, la description de ce que l'on fait au Japon lui paraît fort utile, mais il exprime clairement son scepticisme quant au transfert ou à l'adaptation en Occident de telle ou telle autre intervention pratiquée dans les îles du Soleil Levant. Monsieur Rey partage certaines prises de positions de la thèse. Il est de l'avis qu'il faut changer les politiques actuelles de lutte contre la consommation des drogues, qu'il faut imposer le sevrage obligatoire, la resocialisation et la réinsertion musclée. Il est persuadé qu'il ne faut plus continuer à ergoter sur «Quelles conséquences et interactions résultent de quelles causes sociales, personnelles et politiques?». D'après lui il faut proclamer haut et fort (je le cite): «Dans ce domaine, plus que dans tout autre, les compromis sont sources de division, le toxicomane lui-même est un diviseur et un manipulateur quelle que soit l'origine de sa souffrance. En Suisse, aujourd'hui, il est le seul malade qui dicte à la médecine la prescription des médicaments et des produits qu'il souhaite utiliser. En contrepartie, il lui est demandé de ne pas 'déranger', de ne pas quitter le réseau des soins et de se satisfaire d'être assisté.»

Une telle prise de position relève du domaine de la politique sociale et de la santé publique. La sociologie ne peut pas donner des recettes pour la solution des problèmes sociaux, elle peut, d'une certaine manière, évaluer les conséquences sociales de certains choix politiques et de santé publique. Les choix des solutions appartiennent à la politique. En se cachant derrière les avis des experts, scientifiques et techniques, les politiciens imaginent de réduire les conflits et de délayer la responsabilité des choix. Cette dérive discrédite la politique et les mécanismes démocratiques de régulation.

Je ne discuterai pas les argumentations à propos d'une politique assez contraignante de défense sociale ni cette conception du sociologue en tant que sociothérapeute. Pour moi la crise actuelle de la sociologie dérive précisément de cette prétention à vouloir en faire la médecine de la bonne société, à être la conseilleuse du prince, à être la science infuse des politiques sociales. Fermée cette parenthèse, je voudrais dire, en revanche, les surprises éprouvées en lisant et relisant la deuxième partie de cette thèse. Pourquoi ces surprises?

Les travaux menés en sociologie, et depuis plusieurs lustres, ont mis en jeu ou développé une vaste gamme d'hypothèses et d'analyses sur les processus cognitifs à la base des comportements individuels et collectifs. De l'ethnométhodologie à la philosophie sociale, de la sociologie psychologique à la psychologie sociale en passant par diverses études en sociologie de l'éducation, en sociologie du travail, en sociologie des sciences ou encore en cognition située ou distribuée, les 
thèses abondent sur les modes de formation, d'apprentissage, d'appropriation et de partage des connaissances et des visions du monde. Madame Maurer n'y attache aucune importance. Dans la section intitulée «Vers une théorie sociale du comportement» (feuille 556 et suivantes), il aurait été utile de s'interroger sur les avancées et les apports mutuels, effectifs ou possibles, des recherches menées en sociologie sur ces questions. Je pense, entre autres, aux travaux de Pierre Livet sur les émotions, sur comment celles-ci deviennent des sentiments et pourquoi ceuxci ensuite gouvernent les comportements; je pense aux diverses disciplines liées aux sciences cognitives. L'auteur aurait pu analyser pourquoi et comment les phénomènes sociaux sont déterminés par des «structures» cognitives et dans quelle mesure la vie en société influe sur les formes de cognition (émergence de dispositions, effets d'incorporation des expériences sociales, etc.). Lors de la description du modèle japonais, par exemple, il aurait été précieux de s'interroger sur les capacités cognitives propres aux collectifs, sur la nature des phénomènes sociaux qui relèvent ou non des problèmes de cognition, sur le fait que les mécanismes cognitifs observés hors des laboratoires, à partir des comportements de la vie quotidienne, ne permettent pas de saisir la relation existant entre les conduites individuelles et les croyances collectives. Pourquoi la dynamique du comportement collectif ne renvoie jamais de façon irrésistible aux perceptions, aux motivations et aux attentes des acteurs? Il y a là un écueil sur lequel s'est fracassé jusqu'ici la problématique du changement des comportements, dont il est question dans les feuilles 619 et suivantes de la thèse.

Madame Maurer définit la criminalité, la délinquance et la consommation des drogues «maladies sociales», "pathologies sociales collectives », elles seraient des «déviances pathologiques » à l'origine de la «décadence sociale».

Pour le sociologue la déviance se définit par opposition à la conformité (transgression des normes, des interdits, des obligations, des usages, etc.). La déviance sociologique, déliée de toute codification formelle, se distingue nettement de la délinquance et de la criminalité. Elle peut être positive ou négative, ouvrir les portes aux nouveautés, garantir un certain équilibre social, consolider les identités, raffermir le conformisme. Les interprétations sociologiques du phénomène de la déviance, celles de Merton, Bourdieu, Berger et Luckmann, sont désormais des canons standard en sociologie, de même que celles de la délinquance de Edwin Hardin Sutherland dans le champ juridique. Je ne conteste pas qu'il y ait une déviance naturalisée et une déviance jouée; je ne fait pas ici l'apologie de $\mathrm{H}$. Becker pour lequel la déviance est une catégorie construite dans un processus d'interaction collective qui implique les étiquetés déviants, leur entourage, ceux qui font respecter les normes et ceux qui veulent en imposer des nouvelles. Je veux seulement souligner que le drag-queen, la déviance naturalisée et la déviance jouée restent de cas limites dans le monde social. L'auteur donne au concept de déviance en sociologie une extension qui ne me parait pas convaincante.

Il en est de même de la notion de pathologie. Cette notion du vocabulaire sociologique remonte à Durkheim, pour lequel est normal un phénomène général, pathologique celui qui s'écarte de cette généralité. Dans cette thèse on cite souvent Talcott Parsons, mais de manière inexacte. Parsons, dans The Social System de 1951, fait une distinction entre la réalité organique de la maladie et l'état défini comme maladie par le médecin. C'est cette signification de la santé attribuée par le médecin qu'il a inclus dans son analyse de la réalité sociale. Il dit 
très précisément: «La santé apparaît [...] comme une des conditions requises du fonctionnement de tout système social [...] Un trop haut niveau de maladie, un trop bas niveau de santé apparaissent comme dysfonctionnels par rapport au fonctionnement du système considéré. [...] la maladie nous empêche de nous acquitter de nos rôles sociaux.» Dans aucun cas Parsons parle d'une pathologie au sens médical, d'un état de maladie, d'un écart d'un organe ou d'une fonction du type normal. Pour lui la maladie n'est qu'un des indicateurs des symptômes de désorganisation et de désagrégation. Justement pour cette raison la majorité des sociologues considèrent que la déviance n'est pas une pathologie, que les transgressions sont des faits normaux au sens durkheimien, puisqu'on les constate statistiquement dans toutes les sociétés du passé et du présent. La violation des normes et des règles, pour une partie des individus, est un phénomène inévitable; il est inenvisageable d'empêcher cette violation ainsi qu'il est irréalisable d'éradiquer le crime ou de faire disparaître totalement la consommation des drogues. En plus, les comportements transgressifs, défiants les injonctions ou déjouant les attentes, sont, dans certaines circonstances, des facteurs du changement social.

Encore un mot sur ce que l'auteur dénomme la décadence sociale, laquelle serait la conséquence d'une défense sociale inappropriée ou erronée. Que la décadence puisse hanter nombreux esprits, qu'elle soit au cœur des débats politiques, qu'elle constitue la préoccupation majeure de tous ceux qui craignent la fragilisation des règles, des conventions, l'impuissance des institutions politiques, judiciaires, militaires ou pédagogiques, - rien de plus triviale. Le sociologue, pour sa part, grâce surtout aux études historiques, sait bien que la transgression des règles n'est pas en elle-même un signe de déclin; que la transgression est un phénomène normal et récurrent dans les sociétés humaines, que la décadence reste, du point de vue de la science, un concept d'autant plus ambigu qu'il fait appel à des indicateurs de faits très différents. J'insiste: les comportements déviants sont des phénomènes consubstantiels à la société. Si la violation des règles et des conventions devient une vertu, une attitude ostentatoire répandue dans toutes les couches de la société, un usage courant, alors nous avons le danger de l'Etat policier, la suppression des libertés, la possibilité de réprimer toutes les formes de déviance. C'est bien ainsi qu'on crée les conditions pour le départ des processus de décadence. Une lutte outrancière contre toutes les formes de déviances au nom d'une société idéale, de projets et de théories utopiques, magnanimes et idéalisées, risque de nous rendre aveugles au développement empirique des choses, de nous plonger dans les affres du wébérien paradoxe des conséquences. Les abus ne doivent pas nous faire perdre le sens des libertés, y compris de la liberté de s'égarer et de se tromper. Une société ouverte est une société qui sait qu'il est impossible d'éliminer les transgressions, que la prévention et la répression doivent s'exercer dans le respect de l'autonomie individuelle. Cette société-là reconnaît que les hommes se socialisent dans un contexte historique donné, qu'ils peuvent interpréter les normes déterminant la cohabitation et la vie au sein de la société, qu'ils ont la liberté de s'en inspirer ou non pour donner un sens à leurs actions, à leurs comportements sociaux, qu'ils sont responsables de ce qu'ils font et doivent en assumer les conséquences. Pour s'en convaincre il suffit de lire l'Histoire du déclin et de la chute de l'Empire romain (1776) d'Edward Gibbon, ou Le déclin de l'Occident (1916-1920) d'Oswald Spengler ou encore se référer à Malaise dans la civilisation (1930) de Sigmund Freud. 
Avant de conclure j'aimerais faire encore quelques remarques en marges des feuilles 726-738 intitulées «Pourquoi cette recherche est-elle effectuée dans le cadre de la sociologie?».

Il est devenu courant dans les Universités, faute de vigilance, de mettre sous l'étiquette «sociologie » des produits fort disparates, des horoscopes et de l'astrologie aux variations sur le couple par son linge et les regards des hommes sur les seins nus. Sans parler des expertises-conseils en politique sociale, sur la science, sur l'éducation, sur la lutte à l'alcoolisme, sur la manière d'augmenter le goodwill d'une ville, d'une entreprise ou d'un laboratoire de recherche, ou sur les moyens à adopter pour réaliser le bonheur, l'égalité, la justice sociale.

Il est temps de revenir sur terre et de se souvenir que la sociologie n'est pas l'histoire imaginaire des surlendemains, qu'elle n'est pas une technique de bonne gestion, qu'elle n'est pas la dénonciatrice des maux et des malheurs sociaux. La sociologie n'est ni expertise ni prophétisme, ni intervention thérapeutique ni conseil à la décision. Elle ne dispose d'aucune recette pour soulager les afflictions humaines et les conflits sociaux. La sociologie n'est rien d'autre qu'un corpus de théories ayant comme but d'expliquer les phénomènes sociaux énigmatiques. Si nous donnons une importance primordiale aux mots utilisés, à l'exactitude des connotations et des dénotations des concepts, c'est qu'une théorie sociologique n'est rien d'autre qu' « un ensemble de propositions cohérentes entre elles, dont chacune est acceptable en elle-même, qui forme un appareil déductif, et dont les conséquences sont congruentes avec les phénomènes que l'on peut observer et qui relèvent de son espace de compétence.» (R. Boudon, Y a-t-il encore une sociologie?, p. 128).

Avec d'autres mots, la sociologie est une discipline réflexive, une théorie qui permet de rendre intelligibles les problèmes sociaux mais elle est absolument inapte à fournir des traitements thérapeutiques, à résoudre les problèmes de l'organisation sociale, à dire ce que c'est la bonne société.

En réalité, Madame Maurer ne fait pas de la sociologie; elle plaide, pour une politique de la santé publique, éloignée des enjeux et des dynamiques des luttes pour le pouvoir au sein d'un espace institutionnalisé, et dérivant sa légitimité des sciences sociales. Vision liée à des idéologies, à une conception salvatrice de la sociologie et de l'anthropologie. Vision, par ailleurs, assez en vogue dans les politiques publiques et dans l'action sociale d'aujourd'hui. Mais également chez les praticiens confrontés aux difficultés de voir revenir les mêmes personnes qu'ils avaient déjà traitées.

Je suis convaincu que ce projet, élaborer des orientations en matière de santé publique, est respectable. J'ajoute qu'il s'agit d'un projet plausible, et je n'en doute pas. Mais il doit être énoncé avec plus de netteté, de précision, ne pas être noyé dans un océan de thèmes, dans un catalogue touffu. Afin de bien tracer les contours d'une autre politique de la santé publique, il faudrait élaguer cette thèse, la débarrasser des détails ou des développements inutiles.

Les politiques publiques de la santé sont en friche. Il y a beaucoup de travail à faire, mais sans s'encombrer de bagages inutiles, sans la prétention d'épuiser à jamais le sujet, en reconnaissant qu'il s'agit de domaines relevant davantage de l'art et de la pratique que de la science.

giovanni.busino@bluewin.ch 